\title{
Novel bacterial molybdenum and tungsten enzymes: three-dimensional structure, spectroscopy, and reaction mechanism
}

\author{
Matthias Boll1', Bernhard Schink, ${ }^{2, *}$, Albrecht \\ Messerschmidt ${ }^{3}$ and Peter M.H. Kroneck ${ }^{2}$ \\ ${ }^{1}$ Institut für Biologie II, Universität Freiburg, Schänzlestr. \\ 1, D-79104 Freiburg, Germany \\ ${ }^{2}$ Fachbereich Biologie, Universität Konstanz, D-78457 \\ Konstanz, Germany \\ ${ }^{3}$ Abteilung Strukturforschung, Max-Planck-Institut für \\ Biochemie, Am Klopferspitz 18, D-82152 Martinsried, \\ Germany \\ ${ }^{*}$ Corresponding author \\ e-mail: bernhard.schink@uni-konstanz.de
}

\begin{abstract}
The molybdenum enzymes 4-hydroxybenzoyl-CoA reductase and pyrogallol-phloroglucinol transhydroxylase and the tungsten enzyme acetylene hydratase catalyze reductive dehydroxylation reactions, i.e., transhydroxylation between phenolic residues and the addition of water to a triple bond. Such activities are unusual for this class of enzymes, which carry either a mononuclear Mo or $\mathrm{W}$ center. Crystallization and subsequent structural analysis by high-resolution X-ray crystallography has helped to resolve the reaction centers of these enzymes to a degree that allows us to understand the interaction of the enzyme and the respective substrate(s) in detail, and to develop a concept for the respective reaction mechanism, at least in two cases.
\end{abstract}

Keywords: acetylene hydratase; dithiolene; hydration; 4-hydroxybenzoyl-CoA reductase; pyrogallol-phloroglucinol transhydroxylase; reductive dehydroxylation; transhydroxylation.

\section{Introduction}

Molybdenum enzymes are widespread in nature and have been isolated from green plants, animals and, preferentially, from prokaryotes (Hille, 1996, 2002, 2005; Kisker et al., 1998; Hille et al., 1999; Mendel and Hansch, 2002). Among the latter, anaerobic and facultatively anaerobic bacteria contain such enzymes (Kisker et al., 1998). Molybdenum enzymes are mainly involved in redox reactions, and typically catalyze reactions that have been described as hydroxylations or dehydroxylations. The bacterial molybdenum enzymes catalyze a variety of important reactions in the metabolism of nitrogen- and sulfur-containing compounds, and of various carbonyl compounds, such as aldehydes, formate, CO, and $\mathrm{CO}_{2}$. They have been grouped into various classes, depending on structural properties and sequence similarities of the protein backbone (Hille, 1996; Kisker et al., 1998).

Molybdenum is present as part of a multinuclear cluster with seven iron atoms in nitrogenase, or as part of a MoSCu cluster in carbon monoxide dehydrogenase from Oligotropha carboxydovorans, but in most molybdoenzymes it is found in a single center (Hille, 1996, 2002, 2005; Dobbek and Huber, 2002). In mononuclear molybdoenzymes, the metal is coordinated by one or two equivalents of a unique pyranopterin found only in these and the closely related tungsten-containing enzymes. This pterin derivative has two sulfhydryls from a 1,2-cisdithiolene group, which coordinate the metal (Figure 1). In some bacteria, especially extreme thermophiles, molybdenum is replaced by tungsten in otherwise isofunctional enzymes.

Today, X-ray crystallography allows the resolution of the structures of proteins and their reaction centers at the atomic level. Such structural data can often contribute to an understanding of reaction mechanisms, provided that the functional groups and their linkage to the substrate molecule can be visualized at sufficiently high spatial resolution.

The present contribution does not intend to give a comprehensive overview of all molybdenum enzymes known today. Rather, we focus on structural, spectroscopic and functional aspects of three enzymes that are novel in many respects: (i) the Mo,FeS,FAD enzyme 4hydroxybenzoyl-CoA reductase (HBCR) from Thauera aromatica, a member of the xanthine oxidase family; (ii) the Mo,FeS enzyme pyrogallol-phloroglucinol transhydroxylase (TH) of Pelobacter acidigallici; and (iii) the W,FeS enzyme acetylene hydratase (AH) of Pelobacter acetylenicus. The latter two enzymes belong to the DMSO reductase family (Hille, 1996; Kisker et al., 1998) and do not carry out net redox reactions. TH catalyzes the transfer of hydroxyl groups between phenolic compounds, and $\mathrm{AH}$ adds water to the $\mathrm{C} \equiv \mathrm{C}$ triple bond of acetylene.<smiles>[R]OO[R20](=O)OCC1OC2Nc3nc(N)[nH]c(=O)c3NC2C(SCC)=C1SC</smiles>

Figure 1 Structure of the basic pterin with the dithiolene side chain coordinated to either molybdenum or tungsten (Hille, 1996). 


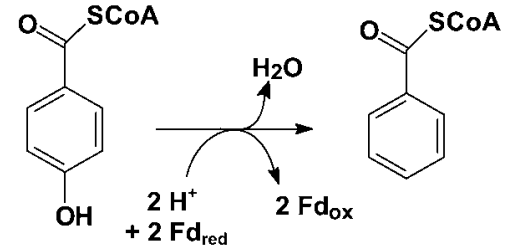

Figure 2 Reaction catalyzed by 4-hydroxybenzoyl-CoA reductase.

\section{4-Hydroxybenzoyl-CoA reductase}

4-Hydroxybenzoyl-CoA reductase is involved in the anaerobic metabolism of aromatic compounds, catalyzing the reductive elimination of the phenolic hydroxyl group from 4-hydroxybenzoyl-CoA to yield benzoyl-CoA and water (Figure 2) (Brackmann and Fuchs, 1993). This reaction plays an important role in the anaerobic degradation of phenol and para-hydroxylated compounds such as para-cresol and 4-hydroxyphenylacetic acid (Schink et al., 2000; Boll and Fuchs, 2005).

$\mathrm{HBCR}$ is a member of the xanthine oxidase family of molybdenum-containing hydroxylases (Gibson et al., 1997). So far, HBCR has only been isolated and characterized from the facultatively anaerobic denitrifying bacterium Thauera aromatica. The 270-kDa enzyme consists of three subunits of 85,35 and $17 \mathrm{kDa}$, resulting in a $(\alpha \beta \gamma)_{2}$ composition (Brackmann and Fuchs, 1993). The large $\alpha$-subunit binds the molybdopterin-cytosinedinucleotide (MCD), the medium $\beta$-subunit harbours one FAD and a [4Fe-4S] cluster, and the small $\gamma$-subunit binds two [2Fe-2S] clusters. Reduced ferredoxin serves as the physiological electron donor (Breese and Fuchs, 1998, Unciuleac et al., 2004b).

The three structural genes of HBCR were originally cloned and sequenced in the anaerobic phototrophic bacterium Rhodopseudomonas palustris (Gibson et al., 1997) and in T. aromatica (Breese and Fuchs, 1998). The structural genes of $\mathrm{HBCR}$ are also present in the genomes of the microaerophilic Magnetospirillum magnetotacticum and of the denitrifying Azoarcus EbN1, both of which are capable of utilizing aromatic com- pounds in the absence of oxygen (Boll, 2005). The amino acid sequences of the three structural subunits are highly similar in these organisms (identities $>60 \%$ ). They also resemble the three subunits of enzymes of the xanthine oxidase family of molybdenum-containing enzymes (approx. 30\% identity). Notably, HBCR is the only member of this family that catalyses a reductive dehydroxylation reaction. The mechanism of this dehydroxylation is discussed in detail in Boll and Fuchs (2005).

\section{Three-dimensional structure and spectroscopy}

Crystals of HBCR obtained in the absence of dioxygen allowed the determination of its three-dimensional structure (Unciuleac et al., 2004a,b). The overall butterfly-like structure of the dimer is similar to that of other members of the xanthine oxidase family (Hille, 2005). Each $\alpha \beta \gamma$ unit carries a complete set of cofactors and is proposed to function as a catalytically independent unit. The relative arrangement of the subunits and the cofactors suggests the following order of electron transfer from the natural electron donor to the substrate: $\mathrm{Fd}_{\text {red }} \rightarrow[4 \mathrm{Fe}-4 \mathrm{~S}] \rightarrow$ $\mathrm{FAD} \rightarrow[2 \mathrm{Fe}-2 \mathrm{~S}] \quad \mathrm{I} \rightarrow$ [2Fe-2S] $\mathrm{II} \rightarrow$ Mo-center $\rightarrow$ substrate (Figure 3). The distances between the redox centers are within the range of biochemical electron-transfer reactions (14 ̊), an exception being the distance of $16.5 \AA$ between the [4Fe-4S] cluster and FAD. However, calculations revealed that electron transfer from the reduced $[4 \mathrm{Fe}-4 \mathrm{~S}]^{1+}$ cluster to oxidized FAD is not rate-limiting in the overall reaction (Unciuleac et al., 2004b).

Iron sulfur clusters In their reduced form, the two $[2 \mathrm{Fe}-2 \mathrm{~S}]^{1+/ 2+}$ clusters of molybdenum hydroxylases typically exhibit distinct EPR signatures: the so-called cluster I shares typical features with $[2 \mathrm{Fe}-2 \mathrm{~S}]^{1+}$ clusters in plant-type ferredoxins with $g_{\mathrm{av}} \approx 1.96$, whereas cluster II exhibits unusual properties, such as highly isotropic $g$ values, broad lines and very fast relaxation behavior (Caldeira et al., 2000). The [2Fe-2S] clusters of HBCR do not fit into this classification. The $g_{\mathrm{av}}$ values of both clusters are greater than 1.96, and neither of the clusters exhibits unusually fast relaxation behavior or a wide spread of $g$ values (Boll et al., 2001). EPR redox titration studies indi-

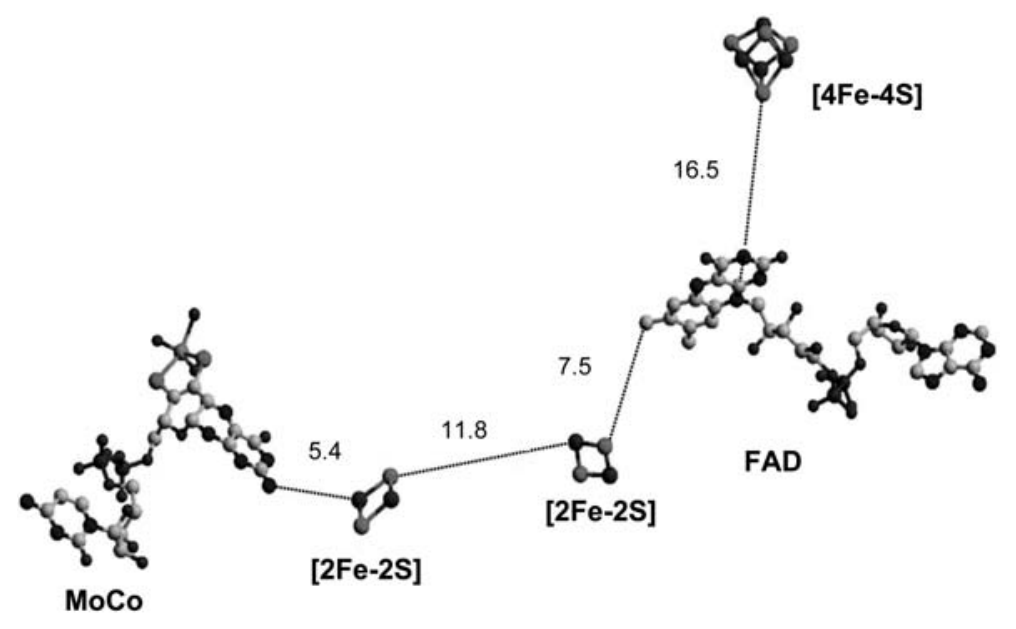

Figure 3 Arrangement of the cofactors in an $\alpha \beta \gamma$-unit of 4-hydroxybenzoyl-CoA reductase. The numbers refer to the minimal distances between the cofactors in $\AA$. 
Table 1 Redox potentials of cofactors of 4hydroxybenzoyl-CoA reductase (Boll et al., 2001).

\begin{tabular}{ll}
\hline Cofactor/redox transition & Redox potential $(\mathrm{mV})$ \\
\hline$[2 \mathrm{Fe}-2 \mathrm{~S}] \mathrm{I}$ & -205 \\
{$[2 \mathrm{Fe}-2 \mathrm{~S}] \mathrm{II}$} & -255 \\
{$[4 \mathrm{Fe}-4 \mathrm{~S}]$} & -465 \\
FAD/FADH & -250 \\
$\mathrm{FADH} / \mathrm{FADH}$ & \\
$\mathrm{Mo}(\mathrm{VI} / \mathrm{Mo}(\mathrm{V})$ & -470 \\
$\mathrm{Mo}(\mathrm{V}) / \mathrm{Mo}(\mathrm{IV})$ & -380 \\
\hline
\end{tabular}

cated that the midpoint redox potentials are within the typical range for [2Fe-2S] clusters and are clearly more positive than that of the [4Fe-4S] cluster (Table 1). Surprisingly, reduction of the [4Fe-4S] cluster affected the EPR properties of the $[2 \mathrm{Fe}-2 \mathrm{~S}]^{1+}$ clusters substantially. At potential values at which the $[4 \mathrm{Fe}-4 \mathrm{~S}]$ clusters were reduced to the paramagnetic state, the spectra of the $[2 \mathrm{Fe}-2 \mathrm{~S}]^{1+}$ clusters gradually disappeared, probably due to broadening or splitting effects (Boll et al., 2001). The unusual EPR properties of the two $[2 \mathrm{Fe}-2 \mathrm{~S}]^{1+}$ clusters are not reflected in an atypical binding mode. In contrast, the small subunit carrying both clusters is the structurally most conserved one between HBCR and other members of the xanthine oxidase family (Unciuleac et al., 2004b). The most pronounced difference between HBCR and all other members of the xanthine oxidase family is the presence of an additional 40-amino-acid polypeptide segment, which is involved in binding the $[4 \mathrm{Fe}-4 \mathrm{~S}]^{1+/ 2+}$ cluster. This additional low-potential iron-sulfur cluster is considered to play an essential role in reversed electron transfer from the donor-reduced ferredoxin to the molybdenum-bound substrate (Unciuleac et al., 2004b).

Flavin cofactor Most molybdenum-containing hydroxylases contain FAD, which is coordinated by the $\beta$-subunit. FAD was also unambiguously identified in HBCR (Breese and Fuchs, 1998). The electrochemical properties of the flavin cofactor are rather unusual. The gap between the two redox transitions (FAD/FADH',

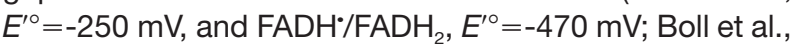
2001 ) is very high (Table 1 ). The role of FAD is predicted to serve as a one-electron carrier between [4Fe-4S] and [2Fe-2S] clusters. This function is rather unusual, as it typically mediates electron transfer between one-electron and two-electron carriers (e.g., NADH).

Molybdenum center As in other members of the xanthine oxidase family, a rapid and resting paramagnetic Mo(V) EPR spectrum can be distinguished in HBCR. The catalytically relevant rapid EPR signal is axial and characterized by $g$ values of 1.990, 1.965 and 1.965 (Boll et al., 2001). Pronounced hyperfine splitting of the signal was observed, which nearly disappeared in $\mathrm{D}_{2} \mathrm{O}$. The electrochemical properties of the Mo center of HBCR are again unusual among members of the xanthine oxidase family: the gap between $\mathrm{Mo}(\mathrm{VI}) /(\mathrm{V})$ and $\mathrm{Mo}(\mathrm{V}) /(\mathrm{IV})$ transitions is exceptionally high $(120 \mathrm{mV})$ and the $\mathrm{Mo}(\mathrm{V}) / \mathrm{Mo}(\mathrm{IV})$ transition has an unusually low redox potential of approximately $-500 \mathrm{mV}$ (Table 1). This low potential is considered to be essential for the proposed first low-potential electron transfer step, yielding a substrate radical anion species (Buckel and Keese, 1995; Boll et al., 2001). The ligands at the Mo atom were identified as two sulfur atoms from the dithiolene functionality of the cofactor, two oxo and one water ligand (Unciuleac et al., 2004b). The latter ligand is considered to cause the observed hyperfine interaction between protons and the paramagnetic $\mathrm{Mo}(\mathrm{V})$ species. Notably, no inorganic sulfur ligand was found, suggesting that the enzyme was crystallized in the desulfo-form, as described first for the crystal structure of aldehyde oxidoreductase (Romão et al., 1995). An additional unusual electron density was found trans to the apical $\mathrm{Mo}=\mathrm{O}$ position. Whether this represents an artifact or an unusual oxygen/sulfur ligand at low occupancy needs to be confirmed by further investigation.

\section{Reaction mechanism}

The large subunit contains a unique $18-\AA$-long and 6 - $\AA$-wide substrate-binding channel that positions the substrate optimally, with its para-hydroxyl functionality towards the deeply buried molybdenum center (Unciuleac et al., 2004b). Replacement of the Mo-bound water ligand by the para-hydroxyl-group has been suggested as the initiation step in the catalytic cycle. In comparison to other molybdenum hydroxylases, HBCR catalyses the reverse reaction, dehydroxylation of the substrate. The mechanism is not a simple reversal of that of xanthine oxidase, as the latter requires a heteroaromatic substrate. Rather, the reaction mechanism suggested for HBCR involves highly reactive radical species, which is atypical for Mo-containing hydroxylases. The unique coenzyme A thiol-ester substrate is considered essential for facilitating the first electron transfer step to the substrate. It is proposed that this facilitates the formation of transient radical species.

\section{Pyrogallol-phloroglucinol transhydroxylase}

Pyrogallol-phloroglucinol transhydroxylase is a key enzyme in the fermentative degradation of gallic acid and pyrogallol by the strictly anaerobic bacterium Pelobacter acidigallici. Gallic acid is first converted to pyrogallol by decarboxylation, and the resulting pyrogallol is isomerized to phloroglucinol by a hydroxyl transfer reaction with 1,2,3,5-tetrahydroxybenzene as a co-substrate or cocatalyst (Brune and Schink, 1990). Migration of the hydroxy group in position 2 of 1,2,3,5-tetrahydroxybenzene to position 5 of pyrogallol forms phloroglucinol and a new molecule of 1,2,3,5-tetrahydroxybenzene, which can enter the reaction cycle again (Figure 4). The enzyme is a heterodimer (molecular mass $133.3 \mathrm{kDa}$ ) consisting of an $\alpha$-subunit (875 amino acids, $100.4 \mathrm{kDa}$ ), and a $\beta$-subunit (274 amino acids, $31.3 \mathrm{kDa}$ ) (Reichenbecher et al., 1994, 1996; Baas and Rétey, 1999). Sequence comparison with other mononuclear molybdenum enzymes suggests that $\mathrm{TH}$ belongs to the dimethylsulfoxide reductase (DMSOR) family (Hille, 1996; Kisker et al., 1998). 


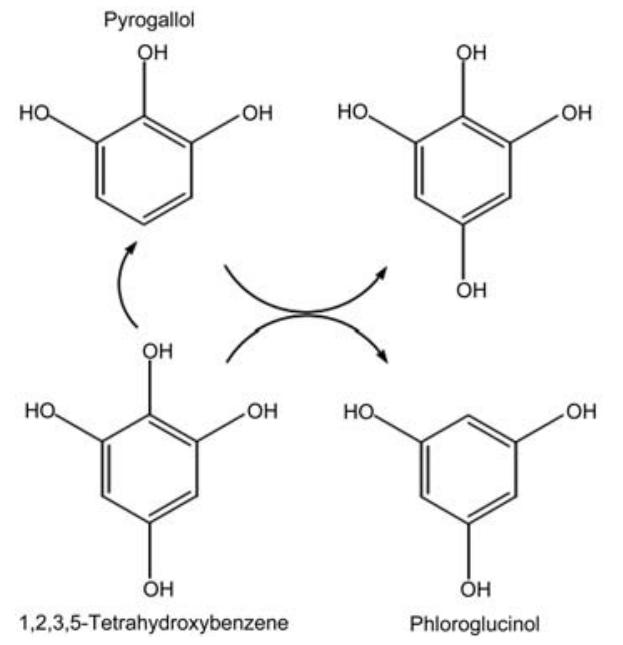

Figure 4 Reaction catalyzed by pyrogallol-phloroglucinol transhydroxylase.

\section{Three-dimensional structure and spectroscopy}

Crystals of $\mathrm{TH}$ in complex with acetate, the substrate pyrogallol, and the inhibitor 1,2,4-trihydroxybenzene could be obtained under reducing conditions in the absence of dioxygen. The native structure was solved by the single anomalous diffraction technique (Messerschmidt et al., 2004).

$\mathrm{TH}$ is a cytoplasmic Mo-enzyme; the $\alpha$-subunit hosts the Mo ion coordinated to four dithiolene sulfurs provided by two molybdopterin-guanine-dinucleotide (MGD) molecules, and the $\beta$-subunit holds three [4Fe-4S] clusters. Members of the DMSOR family share the Mo-containing $\alpha$-subunit, such as DMSOR, formate dehydrogenase $\mathrm{H}(\mathrm{FDH}-\mathrm{H})$, and dissimilatory nitrate reductase (NIR), but may also have one or two additional small subunits, as observed in arsenite oxidase, tungsten-containing formate dehydrogenase $(\mathrm{FDH}-\mathrm{T})(\alpha-$ and $\beta$-subunits), nitrate reductase $A$ (NARGHI) and formate dehydrogenase $\mathrm{N}(\mathrm{FDH}-\mathrm{N})(\alpha-, \beta$ - and $\gamma$-subunits). The only protein ligand to the Mo ion is either serine (DMSOR, $\mathrm{TH}$ ), cysteine (dissimilatory nitrate reductase), aspartate
(NAR A), or selenocysteine (FDH). Arsenite oxidase is unique in having no covalent linkage between the protein and the Mo atom (Ellis et al., 2001).

Overall structure The crystal structure confirms that $\mathrm{TH}$ is a heterodimer, with the $\alpha$ - and $\beta$-subunits consisting of four and three domains, respectively, and the relevant metal and MGD ligands (Figure 5). The four domains are similar to those of other DMSOR family members, organized around the MGD moieties. The fold of $\mathrm{TH}$ is completely different between the secondary structure elements $\beta 3$ and $\alpha 1, \beta 6$ and $\beta 7, \beta 15$ and $\alpha 23$, $\alpha 23$ and $\beta 19, \beta 22$ and $\beta 23, \alpha 35$ and $\beta 25$, as well as $\beta 25$ and $\beta 26$, and involves about 250 amino acid residues. Many of them help to form the substrate and co-substrate binding sites, which are accessible from the solvent through a narrow channel. Domains I and II are ferredoxin-like domains; domain I holds two and domain II one Fe-S cluster. Domain III, starting at residue B190, is folded in a seven-stranded mainly antiparallel $\beta$-barrel. A search with domain III for related 3D structures revealed the same fold for transthyretin (prealbumin) and a closely related one for tenascin (third fibronectin type III repeat). The latter is a cell adhesion protein, and TH may be associated with the cytoplasmic membrane via this domain (Messerschmidt et al., 2004).

Active site The active site of $\mathrm{TH}$ is located in the $\alpha$-subunit and includes the Mo-binding site, with six ligands arranged in a distorted trigonal pyramid. There are four sulfur ligands from both MGD moieties, OG from Ser A175, and an oxygen from acetate, which originates from the crystallization buffer. In the acetate-free native structure, this space is probably filled by a hydroxyl or water molecule. The Mo ion should be in the Mo(IV) oxidation state, as the protein was crystallized under the strict exclusion of dioxygen and the crystallization buffer had an excess of sodium dithionite. The $\mathrm{Mo}(\mathrm{V})$ oxidation state has been detected by EPR (signal at $g_{\mathrm{av}} \approx 1.98$ ) in the enzyme as isolated in the presence of air (Abt, 2001). The side chain of Tyr A560 adopts two different confor-

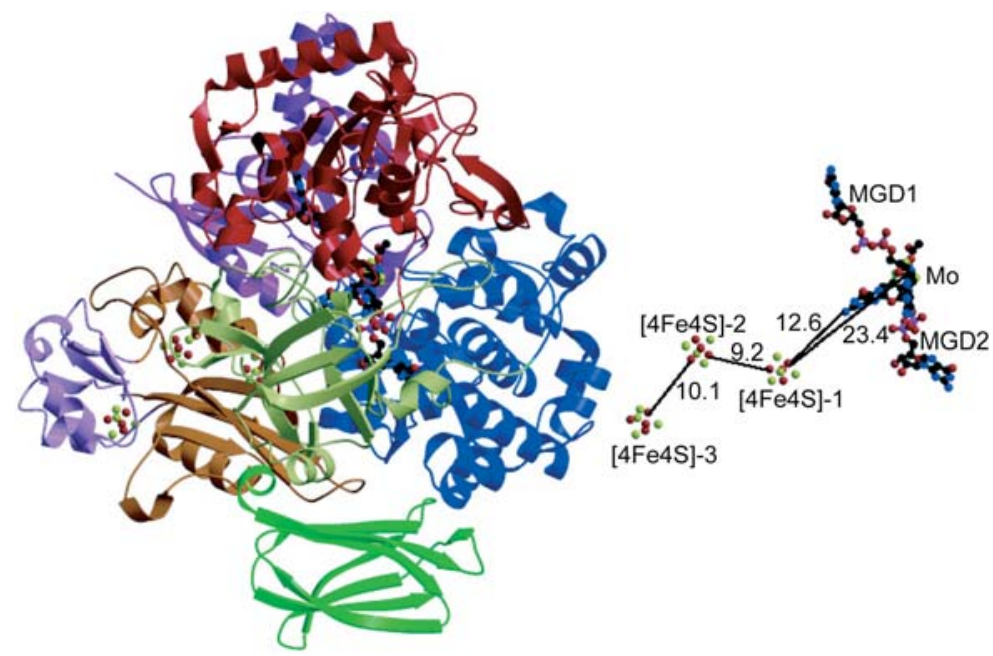

Figure 5 Overall structure of pyrogallol-phloroglucinol transhydroxylase and its cofactors (Messerschmidt et al., 2004). The numbers refer to the minimal distances between the cofactors in $\AA$. 
mations and locks the active site if it is in the right conformation.

Iron sulfur clusters The role of the [4Fe-4S] clusters in the $\beta$-subunit remains unclear. The closest Fe-Fe distances are 10.1 and $9.2 \AA$ (Figure 5), which would be suited to efficient electron transfer. However, the nearest Fe-Mo and Fe-MGD-group distances are 23.4 and $12.6 \AA$. Compared to the distance between $\mathrm{Fe}$ and the pterin cofactor, the distance of $23.4 \AA$ appears rather long for an effective electron transfer between the nearest [4Fe-4S] cluster and the Mo ion. However, the closest distance of $12.4 \AA$ from a [4Fe-4S] cluster to the methyl group C8M of FAD has been found to be functional in adenylylsulfate reductase from Archaeoglobus fulgidus (Fritz et al., 2002). Efficient electron transfer seems to be enhanced here by a strictly conserved tryptophan residue located between the two cofactors and in van der Waals contact with both centers. However, no such aromatic residue between the [4Fe-4S] cluster and the MGD group is found in $\mathrm{TH}$.

The reaction catalyzed by $\mathrm{TH}$ is a net non-redox reaction and does not require external redox equivalents. Therefore, it lacks the [4Fe-4S] cluster in the $\alpha$-subunit, which would allow effective electron transfer between the Mo redox center and the [4Fe-4S] clusters of the $\beta$-subunit, as observed in FDH-T, FDH-N and NARGHI. This might suggest that $\mathrm{TH}$ evolved from such enzymes and carries the $\beta$-subunit as a relict without catalytic function in the $\mathrm{TH}$ reaction, but uses the fibronectin-like domain for membrane association.

\section{Reaction mechanism}

TH does not carry out a net redox reaction, but a hydroxyl transfer between two phenolic substrates. Experiments with ${ }^{18} \mathrm{O}$-labeled water proved that there is no exchange with oxygen from water, and that the hydroxyl group found in the product tetrahydroxybenzene is provided by the co-substrate (Reichenbecher and Schink, 1999). Early on, J. Rétey proposed a detailed mechanism for the transhydroxylase reaction that clearly differed from those reported so far for molybdenum-dependent hydroxylases (Hille et al., 1999). This included a direct hydroxyl transfer from the co-catalyst 1,2,3,5-tetrahydroxy benzene to the o-quinone form of pyrogallol. The oxidation of pyrogallol to the quinone is required for nucleophilic attack by the 2-OH group of the co-catalyst. The molybdenum and [4Fe-4S] centers could be involved in this step. This mechanism leads to $3,4,5,2^{\prime}, 4^{\prime}, 6^{\prime}-$ hexahydroxydiphenylether as intermediate, which was synthesized, as well as its $3,4,5,3^{\prime}, 4^{\prime}, 5^{\prime}$-hexahydroxy isomer. This proposed mechanism was in conflict with the widely expected role of molybdopterin-containing enzymes, according to which the molybdenum center is the acceptor and donor of the transferred $\mathrm{OH}$ or oxo groups (Reichenbecher and Schink, 1999).

Further support in favor of the Rétey mechanism came from X-ray crystallography (Messerschmidt et al., 2004). In the structure of the TH substrate complex, pyrogallol binds with its $\mathrm{O} 1$ oxygen to Mo and replaces the acetate or hydroxyl or water group in the acetate-free enzyme. This reaction is catalyzed by His A144 (NE2 in hydrogen bond distance to $\mathrm{O} 1$ of pyrogallol), which acts as a general base. The other part of the Mo coordination remains unaltered, with similar bond distances as in the native structure. Carbon $\mathrm{C} 1$ of pyrogallol is in the $\mathrm{sp}^{3}$ state, represented by the position of $\mathrm{O} 1$ above the plane of the pyrogallol benzene ring. $\mathrm{O} 2$ is hydrogen-bonded to OE2 of Asp A174 and $\mathrm{O} 3$ to $\mathrm{NH} 2$ of Arg A153. The Mo and the side-chain functions of Asp A174 and Arg A153 are the recognition sites for the substrate. The side chain of Tyr A560 is in the open conformation and allows substrate binding. The space of the alternate conformation has been occupied by water molecules 1-3. The side chains of Tyr A404 and Tyr A152 are situated on top of the pyrogallol molecule. Their phenol rings are stacked parallel to each other. The $\mathrm{OH}$ group of Tyr A404 and the SG of Cys A557 are within hydrogen bonding distance of C5 of pyrogallol and may play a role as a general base in the catalysis of hydroxyl transfer from the co-substrate to the substrate. The space below the benzene ring of pyrogallol is lined by hydrophobic residues, such as Trp A176, Trp A354 and Phe A468. They create the hydrophobic surface region for binding of the hydrophobic part of the substrate molecule in the active site.

The crystal structure of the TH inhibitor complex shows the 1,2,4-trihydroxybenzene molecule bound with its 05 atom coordinated to the Mo. The other part of the Mo coordination is identical to the native structure of the $\mathrm{TH}$ pyrogallol complex, with similar bond distances. The side chain of Tyr A560 is in the closed conformation and the $\mathrm{OH}$ is bound to $\mathrm{O} 2$ of the inhibitor. $\mathrm{O} 4$ is hydrogen-bonded to Asp A174 and 01 points in the direction of the side chain of Cys A577. Arg A153 cannot contact the inhibitor molecule because the corresponding $\mathrm{OH}$-function is missing in the inhibitor.

Three different proposals for the catalytic mechanism of $\mathrm{TH}$ had been made before the crystallographic information became available (Hille et al., 1999). The Rétey mechanism includes 1,2,3,5-tetrahydroxybenzene as cosubstrate through a diphenylether intermediate, and the transferred hydroxyl does not originate from the solvent. Note that the other two mechanisms function without a co-substrate and involve a rotation of the substrate in the active site during catalysis, and the hydroxyl stems from the solvent (Hille et al., 1999) The three-dimensional structure of $\mathrm{TH}$ supports the participation of a co-substrate in the mechanism. Figure 6 shows a schematic view of the active site with bound pyrogallol, as found in the relevant complex structure and manually docked cocatalyst. It adopts the position of the phenyl ring of Tyr A560 when it is in the closed conformation and can form several hydrogen bonds with the protein and pyrogallol, indicated by dashed lines (Figure 6). O2, the hydroxyl to be transferred to C5 of pyrogallol, is in close proximity to this atom. Tyr A404 is the most probable candidate to act as the general base in this transfer reaction, because its $\mathrm{OH}$-group lies at appropriate distances from both atoms, but Cys A557 could also play this role. This mechanism is in line with the experimental findings that 1,2,3,5-tetrahydroxybenzene is needed to start the reaction and that the transferred hydroxyl does not come from the solvent. Based on the structural data, the proposed mechanism involves Asp A174, His A144, Tyr 


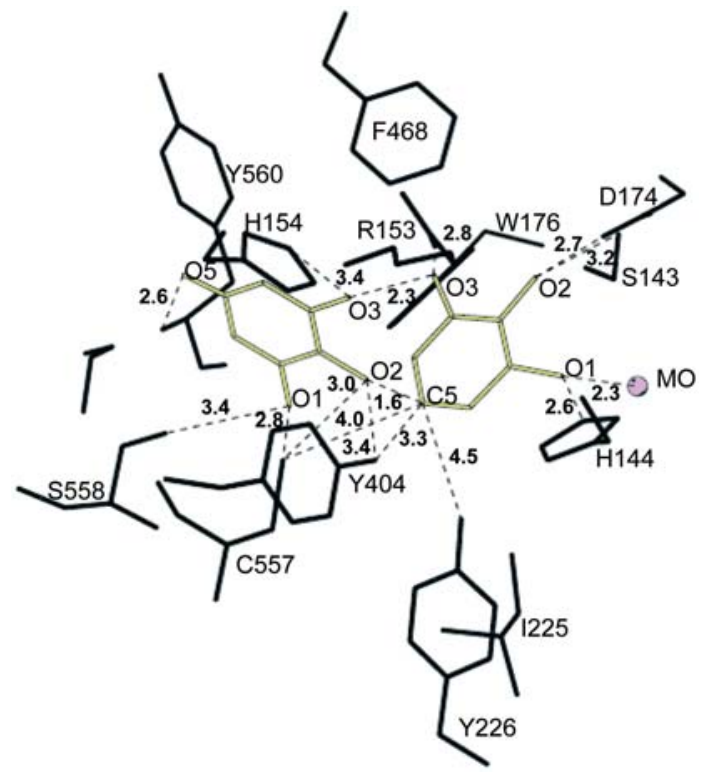

Figure 6 Schematic representation of the active site of pyrogallol-phloroglucinol transhydroxylase, with bound pyrogallol and manually docked co-catalyst 1,2,3,5-tetrahydroxybenzene (Messerschmidt et al., 2004).

A404 and the Mo as catalytic residues and goes through reaction intermediates as proposed by Rétey (Hille et al., 1999; Messerschmidt et al., 2004). Pyrogallol enters the active site first and is bound, as observed in the TH pyrogallol complex structure. The molybdenum is in the $+\mathrm{VI}$ oxidation state, and the residues Asp A174, Tyr A404 and His A144 are deprotonated. His A144 abstracts the proton from $\mathrm{O} 1$, thus promoting its binding to $\mathrm{Mo}(\mathrm{VI})$. Molybdenum oxidizes the enol tautomer of pyrogallol to the ortho-quinone form and is reduced to Mo(IV). Tyr A404 abstracts a proton from $\mathrm{O} 2$. Subsequently, the $\mathrm{O}^{-}$of the co-substrate attacks the C5 of pyrogallol in a nucleophilic manner. A bridging bond from $\mathrm{O} 2$ to $\mathrm{C} 5$ of the pyrogallol is formed, causing the flip of one double bond in the ring system and nucleophilic attack of the double bond between $\mathrm{C} 3$ and $\mathrm{O} 3$ of pyrogallol at the proton of NE2 of $\mathrm{His} \mathrm{A} 144$. The rearrangements indicated lead to the formation of a diphenylether, as suggested earlier. Hereafter, the covalent adduct between substrate and co-substrate can be cleaved to form the product phloroglucinol and the quinone form of tetrahydroxybenzene. Reduction of the latter by $\mathrm{Mo}(\mathrm{IV})$ and transfer of the respective protons from Asp A174 and Tyr A404 close the catalytic cycle (Messerschmidt et al., 2004).

\section{Acetylene hydratase}

To date, acetylene is the only hydrocarbon known to be metabolized in the absence and presence of molecular oxygen in the same manner. Acetylene hydratase $(\mathrm{AH})$ is the first enzyme involved in the fermentative conversion of acetylene to acetate and ethanol by the strictly anaerobic fermenting bacterium $P$. acetylenicus (Schink, 1985). Growth of $P$. acetylenicus with acetylene depends on the supply of tungstate or, to a lower degree, of molybdate in the medium. $\mathrm{AH}$ converts acetylene to acetaldehyde, a reaction distinct from the conversion of acetylene to ethylene by nitrogenase (Burgess and Lowe, 1996). The enzyme was purified as a monomer ( $85 \mathrm{kDa})$. The $\mathrm{N}$-terminus of the protein shows a typical binding motif for an iron-sulfur cluster, of the type Cys- $\mathrm{X}_{2}-\mathrm{Cys}_{-} \mathrm{X}_{3}$-Cys (Rosner and Schink, 1995). Phylogenetic analyses, based on the amino acid sequence, revealed that acetylene hydratase belongs to the DMSOR family (Abt, 2001).

Highly active $\mathrm{W}$-enzyme $\left(42.3 \mathrm{U} / \mathrm{mg}, 50^{\circ} \mathrm{C}\right)$ contained $3.5 \mathrm{~mol}$ iron and $1.1 \mathrm{~mol}$ tungsten $/ \mathrm{mol}$ enzyme, whereas molybdenum and selenium were absent (Abt, 2001). The specific activity of the enzyme peaks between $\mathrm{pH} 6.0$ and 7.0 (temperature optimum at $50^{\circ} \mathrm{C}$ ). Although the addition of water to the $\mathrm{C} \equiv \mathrm{C}$ triple bond does not represent a net redox reaction, a strong reductant such as $\mathrm{Ti}(\mathrm{III})$-citrate or dithionite is required to observe activity (Meckenstock et al., 1999).

$\mathrm{HC} \equiv \mathrm{CH}+\mathrm{H}_{2} \mathrm{O} \rightarrow\left[\mathrm{H}_{2} \mathrm{C}=\mathrm{C}(\mathrm{OH}) \mathrm{H}\right] \rightarrow \mathrm{CH}_{3} \mathrm{CHO}$

ICP/MS, EPR, and UV/Vis-spectroscopy revealed that $P$. acetylenicus is able to insert molybdenum into the bisMGD cofactor of acetylene hydratase. The Mo-isoenzyme contained $3.1 \mathrm{~mol}$ iron, $0.5 \mathrm{~mol}$ molybdenum, and practically no tungsten per mol enzyme. The specific activity $\left(16.7 \mathrm{U} / \mathrm{mg}, 50^{\circ} \mathrm{C}\right)$ was significantly lower than the specific activity of the W-enzyme. A vanadium-containing acetylene hydratase was not obtained. The purified enzyme from the corresponding vanadate cultivation contained practically no vanadium and only small amounts of tungsten and molybdenum (each $\sim 0.05$ $\mathrm{mol} / \mathrm{mol}$ acetylene hydratase). The specific activity of this enzyme preparation was very low $\left(2.6 \mathrm{U} / \mathrm{mg}, 50^{\circ} \mathrm{C}\right)(\mathrm{Abt}$, 2001).

\section{Structure and spectroscopy}

Crystals of W-acetylene hydratase were obtained both in the presence and absence of dioxygen. Crystals grown under the exclusion of dioxygen in the presence of $\mathrm{Ti}(\mathrm{III})$ citrate or dithionite diffracted to a resolution better than $2.5 \AA$ (Einsle et al., 2005). Based on sequence homologies to the structures available for molybdenum/tungsten hydroxylases, the structure of the tungsten-containing formate dehydrogenase from Desulfovibrio gigas (Raaijmakers et al., 2002) was chosen as a starting point for the construction of a first model of acetylene hydratase.

According to EPR spectroscopy, $\mathrm{AH}$ contains a single [4Fe-4S] cluster with a midpoint redox potential of $-410 \pm 20 \mathrm{mV}$. Preparation of the enzyme under air led to a mutated enzyme with a [3Fe-4S] cluster, whereas preparation under reducing conditions maintained the [4Fe4S] cluster (Meckenstock et al., 1999). The enzyme activity was maximum under strictly reducing conditions and exhibited $50 \%$ of maximum activity at $E_{0}{ }^{\prime}=-340 \pm$ $20 \mathrm{mV}$. [Fe'"'(CN) $\left.{ }_{6}\right]^{3-}$-oxidized AH exhibited resonances of a $W(V)$ center, whereas the enzyme from molybdate (95Mo)-cultivated cells showed resonances of a ${ }^{95} \mathrm{Mo}(\mathrm{V})$ center (Abt, 2001). The UV/Vis spectrum of AH (as isolated) showed broad absorption shoulders resulting from the FeS cluster around $400 \mathrm{~nm}$, and a broad absorption maximum around $600 \mathrm{~nm}$ from $\mathrm{S} \rightarrow \mathrm{W}$ charge-transfer transitions. 


\section{Reaction mechanism}

$\mathrm{AH}$ has to be activated by a strong reductant such as $\mathrm{Ti}(\mathrm{III})$-citrate or dithionite to achieve full activity. Under these conditions, the ferredoxin-type iron-sulfur center is in its reduced $[4 \mathrm{Fe}-4 \mathrm{~S}]^{1+}$ state, and tungsten is in the tetravalent oxidation state (Meckenstock et al., 1999). Oxic preparations of $\mathrm{AH}$ possess a [3Fe-4S] cluster, but exhibit similar specific enzyme activity compared to the anoxic preparation carrying the intact [4Fe-4S] cluster (Meckenstock et al., 1999). Redox titrations of the ironsulfur center and of the enzyme activity gave potentials of -410 and $-340 \mathrm{mV}$, respectively. Thus, acetylene hydratase is active when the iron-sulfur center is still oxidized in the $[4 \mathrm{Fe}-4 \mathrm{~S}]^{2+}$ state. Setting the potential to $\leq-410 \mathrm{mV}$ brought the iron-sulfur center to the [4Fe$4 S]^{1+}$ state, but did not change the activity of the enzyme. Model studies demonstrated the likely participation of a W(IV) site in the catalysis of the hydration of acetylene, whereas the corresponding $\mathrm{W}(\mathrm{VI})$ remained inactive (Yadav et al., 1997).

In addition to the classical $\mathrm{Hg}(\mathrm{II}) / \mathrm{H}^{+}$-catalyzed Markownikoff hydration of alkynes, numerous transition metal catalysts have been developed for the synthesis of ketones via hydration of alkynes, including the anti-Markownikoff hydration of terminal alkynes catalyzed by a $\mathrm{Ru}(\mathrm{II})$ /phosphane mixture (Tokunaga and Wakatsuki, 1998). A similar addition/elimination process might be envisaged for the hydration of acetylene, with a vinyltungsten intermediate that could undergo direct insertion of $\mathrm{OH}$ to form the vinyl alcohol and hence acetaldehyde (Abt, 2001).

Similar to the Mo,FeS enzyme nitrogenase, which catalyzes the reduction of $\mathrm{N}_{2}$ to ammonia, and which also reacts with other triply bonded substrates such as acetylene, the binding mode of acetylene and its site of binding in $\mathrm{AH}$ remain unknown. For nitrogenase, considerations of model organometallic compounds and computational results have led to a number of substratebinding models, some involving one or more $\mathrm{Fe}$ atoms located in the central portion of the FeMo-cofactor, others favoring the Mo atom. With propargyl alcohol, $\mathrm{HC} \equiv \mathrm{C}-\mathrm{CH}_{2} \mathrm{OH}$, a novel bio-organometallic intermediate of nitrogenase could be identified in which a metallacyclopropane ring is bound to a single Fe atom of the FeMo-cofactor (Lee et al., 2004). In preliminary experiments, $\mathrm{AH}$ appears to react with propargylalcohol to an as yet unidentified product. Note that $\mathrm{CO}$ and NO impaired the activity of $\mathrm{AH}$ significantly, and both acetylene mono- and dicarboxylate were not hydrated in cell extracts of $P$. acetylenicus (Rosner and Schink, 1995).

\section{Outlook}

4-Hydroxybenzoyl-CoA reductase, an unusual member of the xanthine oxidase family, has been characterized in detail with respect to its biochemistry, spectroscopy and three-dimensional structure. However, several important questions still remain unanswered. The coordination of Mo has not yet been fully elucidated, and whether the electron density detected in a trans position to the apical $\mathrm{Mo}=\mathrm{O}$ represents a novel Mo ligation mode needs fur- ther investigations. So far, little is known about the reaction mechanism of HBCR. In particular, additional experimental evidence for the proposed mechanism involving a radical intermediate is required. Finally, the question emerges as to what type of enzymes are involved in related reductive reactions of anaerobic aromatic metabolism, e.g., the proposed reductive dehydroxylation of 2-hydroxybenzoyl-CoA to benzoyl-CoA (Bonting and Fuchs, 1996) and the reductive deamination of 4-aminobenzoyl-CoA to benzoyl-CoA (Schnell and Schink, 1991).

The structure of pyrogallol-phloroglucinol TH and complexes with substrate and inhibitor has been solved at high resolution, but open questions remain with respect to the reaction mechanism. In earlier studies it was speculated that the iron-sulfur centers in this enzyme could be involved in reductive and oxidative modification of the phenolic substrates to allow for hydroxyl transfer from one ring to the other. In view of the three-dimensional structure reported here, this assumption is unlikely. Still, it remains unclear how this hydroxyl transfer can be accomplished. Of special interest in this context is the question as to whether the molybdopterin-bound Mo undergoes a redox change during the reaction, or whether it only acts as a structural factor to position the two substrate molecules. A pterin radical that was discovered earlier in bacterial aldehyde dehydrogenases (Luykx et al., 1998) was not detected in TH. Ongoing mechanistic investigations by Paizs and Rétey on the formation and decay of diphenylether intermediates during turnover of $\mathrm{TH}$ will hopefully answer some of the open questions in the near future.

For the W,FeS enzyme acetylene hydratase, which can be compared to some extent to the Mo,FeS enzyme nitrogenase, the mode and site of substrate binding, as well as the reaction mechanism, remain enigmatic. So far, structural studies have not helped much in resolving this problem because the substrate is extremely small and difficult to handle. In this case, X-ray crystallography after binding acetylene to $\mathrm{AH}$ under high pressure, or binding of substrate analogues such as propargyl alcohol, appear to be promising perspectives for a better understanding of the reaction mechanism of this unusual enzyme.

\section{Acknowledgments}

Financial support by Deutsche Forschungsgemeinschaft (SPP 1071) is gratefully acknowledged. The authors thank the past and present members of their groups, mentioned in the cited references, for the many important contributions to the success of the DFG priority program 'Radicals in Enzymatic Catalysis'.

\section{References}

Abt, D. (2001). Tungsten-acetylene hydratase from Pelobacter acetylenicus and molybdenum-transhydroxylase from Pelobacter acidigallici: two novel molybdopterin and iron-sulfur containing enzymes. Ph.D. Thesis, University of Konstanz, Germany.

Baas, D. and Rétey, J. (1999). Cloning, sequencing and heterologous expression of pyrogallol-phloroglucinol transhydroxylase from Pelobacter acidigallici. Eur. J. Biochem. 265, 896-901. 
Boll, M. (2005). Key enzymes in the anaerobic aromatic metabolism catalysing Birch-like reductions. Biochim. Biophys. Acta 1707, 34-50.

Boll, M. and Fuchs, G. (2005). Unusual reactions involved in anaerobic metabolism of phenolic compounds. Biol. Chem. 386, 989-997.

Boll, M., Fuchs, G., Meier, C., Trautwein, A.X., El Kasmi, A., Ragsdale, S.W., Buchanan, G., and Lowe, D.J. (2001). Redox centers of 4-hydroxybenzoyl-CoA reductase, a member of the xanthine oxidase family of molybdenum-containing enzymes. J. Biol. Chem. 276, 47853-47862.

Bonting, C.F. and Fuchs, G. (1996). Anaerobic metabolism of 2hydroxybenzoic acid (salicylic acid) by a denitrifying bacterium. Arch. Microbiol. 165, 402-808.

Brackmann, R. and Fuchs, G. (1993). Enzymes of anaerobic metabolism of phenolic compounds. 4-Hydroxybenzoyl-CoA reductase (dehydroxylating) from a denitrifying Pseudomonas species. Eur. J. Biochem. 213, 563-571.

Breese, K. and Fuchs, G. (1998). 4-Hydroxybenzoyl-CoA reductase (dehydroxylating) from the denitrifying bacterium Thauera aromatica. Prosthetic groups, electron donor, and genes of a member of the molybdenum-flavin-iron-sulfur proteins. Eur. J. Biochem. 251, 916-923.

Brune, A. and Schink, B. (1990). Pyrogallol-to-phloroglucinol conversion and other hydroxyl-transfer reactions catalyzed by cell extracts of Pelobacter acidigallici. J. Bacteriol. 172, 1070-1076.

Buckel, W. and Keese, R. (1995). One-electron reactions of CoASH esters in anaerobic bacteria. Angew. Chem., Int. Ed. 34, 1502-1506.

Burgess, B.K. and Lowe, D.L. (1996). Mechanism of molybdenum nitrogenase. Chem. Rev. 96, 2983-3011.

Caldeira, J., Belle, V., Asso, M., Guigliarelli, B., Moura, I., Moura, J.J.G., and Bertrand, P. (2000). Analysis of the electron paramagnetic resonance properties of the $[2 \mathrm{Fe}-2 \mathrm{~S}]^{1+}$ centers in molybdenum enzymes of the xanthine oxidase family: assignment of signals I and II. Biochemistry 39, 2700-2707.

Dobbek, H. and Huber, R. (2002). The molybdenum and tungsten cofactors: a crystallographic view. In: Metals in Biological Systems, Vol. 39, A. Sigel and H. Sigel, eds. (New York, Basel: Marcel Dekker), pp. 227-263.

Einsle, O., Niessen, H., Abt, D.J., Seiffert, G., Schink, B., Huber, R., Messerschmidt, A., and Kroneck, P.M.H. (2005). Crystallization and preliminary X-ray analysis of the tungstendependent acetylene hydratase from Pelobacter acetylenicus. Acta Crystallogr. F61, 299-301.

Ellis, P.J., Conrads, T., Hille, R., and Kuhn, P. (2001). Crystal structure of the $100 \mathrm{kDa}$ arsenite oxidase from Alcaligenes faecalis in two crystal forms at $1.64 \AA$ and $2.03 \AA$. Structure 9, 125-132.

Fritz, G., Roth, A., Schiffer, A., Büchert, T., Bourenkov, G., Bartunik, H.D., Huber, H., Stetter, K.O., Kroneck, P.M.H., and Ermler, U. (2002). Structure of adenylylsulfate reductase from the hyperthermophilic Archaeoglobus fulgidus at $1.6 \AA$ resolution. Proc. Natl. Acad. Sci. USA 99, 1836-1841.

Gibson, J., Dispensa, M., and Harwood, C.S. (1997). 4-Hydroxybenzoyl coenzyme A reductase (dehydroxylating) is required for anaerobic degradation of 4-hydroxybenzoate by Rhodopseudomonas palustris and shares features with molybdenum-containing hydroxylases. J. Bacteriol. 179, 634-642.

Hille, R. (1996). The mononuclear molybdenum enzymes. Chem. Rev. 96, 2757-2816.

Hille, R. (2002). Molybdenum and tungsten in biology. Trends Biochem. Sci. 27, 360-367.

Hille, R. (2005). Molybdenum-containing hydroxylases. Arch. Biochem. Biophys. 433, 107-116.

Hille, R., Rétey, J., Bartlewski-Hof, U., Reichenbecher, W., and Schink, B. (1999). Mechanistic aspects of molybdenum-containing enzymes. FEMS Microbiol. Rev. 22, 489-501.

Kisker, C., Schindelin, H., Baas, D., Retey, J., Meckenstock,
R.U., and Kroneck P.M. (1998). A structural comparison of molybdenum cofactor-containing enzymes. FEMS Microbiol. Rev. 22, 503-521.

Lee, H.-I., Igarashi, R.Y., Laryukhin, M., Doan, P.E., Dos Santos, P.C., Dean, D.R., Seefeldt, L.C., and Hoffmann, B.M. (2004). An organometallic intermediate during alkyne reduction by nitrogenase, J. Am. Chem. Soc. 126, 9563-9569.

Luykx, D.M.A., Duine, J.A., and de Vries, S. (1998). Molybdopterin radical in bacterial aldehyde dehydrogenases. Biochemistry $37,11366-11375$.

Meckenstock, R.U., Krieger, R., Ensign, S., Kroneck, P.M.H., and Schink, B. (1999). Acetylene hydratase of Pelobacter acetylenicus: molecular and spectroscopic properties of the tungsten iron-sulfur enzyme. Eur. J. Biochem. 264, 176-182.

Mendel, R.R. and Hansch, R. (2002). Molybdoenzymes and molybdenum cofactor in plants. J. Exp. Bot. 53, 1689-1698.

Messerschmidt, A., Niessen, H., Abt, D., Einsle, O., Schink, B., and Kroneck, P.H.M. (2004). Crystal structure of pyrogallolphloroglucinol transhydroxylase, a Mo enzyme capable of intermolecular hydroxyl transfer between phenols. Proc. Natl. Acad. Sci. USA 101, 11571-11576.

Raaijmakers, H., Maciera, S., Dias, J.M., Teixeira, S., Bursakov, S., Huber, R., Moura, J.J., Moura, I., and Romao, M.J. (2002). Gene sequence and the $1.8 \AA$ crystal structure of the tungsten-containing formate dehydrogenase from Desulfovibrio gigas. Structure 10, 1261-1272.

Reichenbecher, W. and Schink, B. (1999). Towards the reaction mechanism of pyrogallol-phloroglucinol transhydroxylase of Pelobacter acidigallici. Biochim. Biophys. Acta 1430, 245-253.

Reichenbecher, W., Brune, A., and Schink, B. (1994). Transhydroxylase of Pelobacter acidigallici: a molybdoenzyme catalyzing the conversion of pyrogallol to phloroglucinol. Biochim. Biophys. Acta 1204, 217-224.

Reichenbecher, W., Rüdiger, A., Kroneck, P.M.H., and Schink, B. (1996). One molecule of molybdopterin guanine dinucleotide is associated with each subunit of the heterodimeric Mo-Fe$S$ protein transhydroxylase of Pelobacter acidigallici as determined by SDS/PAGE and mass spectrometry. Eur. J. Biochem. 237, 406-413.

Romão, M.J., Archer, M., Moura, I., Moura, J.J.G., LeGall, J., Schneider, M., Hof, P., and Huber, R. (1995). Crystal structure of the xanthine oxidase-related aldehyde oxidoreductase from $D$. gigas. Science 270, 1170-1176.

Rosner, B. and Schink, B. (1995). Purification and characterization of acetylene hydratase of Pelobacter acetylenicus, a tungsten iron-sulfur protein. J. Bacteriol. 177, 5767-5772.

Schink, B. (1985). Fermentation of acetylene by an obligate anaerobe, Pelobacter acetylenicus sp. nov. Arch. Microbiol. 142, 295-301.

Schink, B., Philipp, B., and Müller, J. (2000). Anaerobic degradation of phenolic compounds. Naturwissenschaften 87 , 12-23.

Schnell, S. and Schink, B. (1991). Anaerobic aniline degradation via reductive deamination of 4-aminobenzoyl CoA in Desulfobacterium anilini. Arch. Microbiol. 155, 183-190.

Tokunaga, M. and Wakatsuki, Y. (1998). The first anti-Markownikov hydration of terminal alkynes: formation of aldehydes catalyzed by a ruthenium(II)/phosphane mixture. Angew. Chem. Int. Ed. 37, 2867-2869.

Unciuleac, M., Boll, M., Warkentin, E., and Ermler, U. (2004a). Crystallization of 4-hydroxybenzoyl-CoA reductase and the structure of its electron donor ferredoxin. Acta Crystallogr. D. Biol. Crystallogr. 60, 388-389.

Unciuleac, M., Warkentin, E., Page, C.C., Boll, M., and Ermler, U. (2004b). Structure of a xanthine oxidase-related 4hydroxybenzoyl-CoA reductase with an additional [4Fe-4S] cluster and an inverted electron flow. Structure 12, 2249-2256.

Yadav, J., Damar, K.D., and Sabyasachi, S. (1997). A functional mimic of the new class of tungstoenzyme, acetylene hydratase. J. Am. Chem. Soc. 119, 4315-4316. 